\title{
CDISC SDTM Specimen Type Terminology
}

National Cancer Institute

\section{Source}

National Cancer Institute. CDISC SDTM Specimen Type Terminology. NCI Thesaurus.

Code C78734.

Terminology associated with the specimen type codelist of the Clinical Data Interchange Standards Consortium (CDISC) Study Data Tabulation Model (SDT M). 